\title{
SUPPORTING GROUP COGNITION, INDIVIDUAL LEARNING and Community Practices in Dynamic Geometry
}

\author{
Gerry Stahl \\ The Math Forum and the iSchool \\ Drexel University \\ Philadelphia, USA \\ Gerry@GerryStahl.net
}

\begin{abstract}
Group cognition is analyzed at the small-group unit of analysis. It involves the semantics, syntactics and pragmatics of natural language, gestures, inscriptions, etc. The meaningmaking processes involve inputs from individuals, based on their interpretation of the on-going context. They are also responses to the on-going social/historical/cultural/linguistic context, which they can reproduce and modify. Technologies play a central role in mediating the multi-level, intertwined processes. Emergent technologies should be designed to support this mediation. Collaboration environments should be designed to prepare groups, individuals and communities to take advantage of the technical functionality and to promote learning at all levels. This paper reports on the design of a curriculum in dynamic geometry to support group cognition, individual learning and community practices in a coordinated way.
\end{abstract}

Keywords-Computer-supported collaborative learning; group cognition; math discourse, dynamic geometry

\section{INTRODUCTION}

Group cognition is analyzed at the small-group unit of analysis. It involves the semantics, syntactics and pragmatics of natural language, gestures, inscriptions, etc. The meaningmaking processes involve inputs from individuals, based on their interpretation of the on-going context (Stahl, 2006, esp. Ch. 16). They also take into account the larger social/historical/cultural/linguistic context, which they can reproduce and modify (Stahl, 2013). Applying this perspective to the learning of mathematics, we adopt a discourse-centered view of mathematical understanding as the ability to engage in significant mathematical discussion (Sfard, 2008; Stahl, 2008). Here, "discourse" includes gesture, inscription, representation and symbol, as well as speech and text; these are often closely interwoven in effective interactions (Çakır \& Stahl, 2012; Çakir, Zemel \& Stahl, 2009).

Technologies play a central role in mediating the multilevel, intertwined problem-solving, learning and knowledgebuilding processes. Emergent technologies should be designed to support this mediation. This involves considering within the design process of collaboration environments how to prepare groups, individuals and communities to take advantage of the designed functionality and to promote mathematical thinking at all levels. This paper reports on the design of a curriculum in dynamic geometry to support group cognition, individual learning and community practices in a coordinated way.

We have been developing a collaboration environment for small groups of students to explore mathematics especially dynamic geometry - together online (Stahl, 2009). Our Virtual Math Teams (VMT) environment now includes a multi-user version of GeoGebra, an open-source dynamicgeometry tool (Stahl et al., 2010). Shared chat rooms in this VMT environment can include:

- Personal GeoGebra tabs for an individual to experiment with dynamic-geometry explorations and constructions.

- Group GeoGebra tabs for a team of students to experiment together with dynamic-geometry explorations and constructions.

- A text-chat window for a team to discuss its collaborative explorations, while it is working together or to ask questions when team members have problems in their individual work.

- A shared whiteboard and a group wiki page for the group to summarize its findings.

- The wiki can be used by a whole class or a community of teams to view and comment on what each team has accomplished.

- Logs of the text chat and a replayer, which allows anyone to replay a collaboration session in complete detail for purposes of reflection and/or analysis.

We have conducted pilot trials of the VMT-withGeoGebra environment and have found that this relatively complex system requires some preparation and training for students, student groups and classes to use effectively without encountering frustration. In response to issues identified in the analysis of the multi-user GeoGebra use sessions, we have drafted a set of dynamic-geometry curricular activities, interspersed with tutorial tours of the technology features (Stahl, 2012a). These materials are designed for use both by teachers in professionaldevelopment contexts and by students in online-classroom or after-school settings.

The curriculum activities have been designed to promote collaborative learning, particularly as it occurs in significant mathematical discourse about geometry. Collaborative learning involves a subtle interplay of processes at the individual, small-group and classroom levels of engagement, cognition and reflection. Accordingly, the activities are 
structured with sections for individual work, small-group collaboration and whole-class discussion. It is hoped that this mixture will enhance motivation, extend attention and spread understanding.

\section{CURRICULAR GOALS}

The goal of our set of activities is to improve the following skills in math teachers and their students:

1. To engage in significant mathematical discourse; to collaborate on and discuss mathematical activities in supportive small online groups.

2. To collaboratively explore mathematical phenomena and dependencies; to make mathematical phenomena visual in multiple representations; and to vary their parameters.

3. To construct mathematical diagrams - understanding and exploring their structural dependencies.

4. To notice, wonder about and form conjectures about mathematical relationships; to justify, explain and prove mathematical findings.

5. To understand core concepts, relationships, theorems and constructions of basic high-school geometry.

The working hypothesis of the activities is that these goals can be furthered through an effective combination of:

1. Collaborative experiences in mathematical activities with guidance in collaborative, mathematical and accountable geometric discourse.

2. Exploring dynamic-mathematical diagrams and multiple representations.

3. Designing dependencies in dynamic-mathematical constructions.

4. Explaining conjectures, justifications and proofs.

5. Engagement in well-designed activities around basic high-school geometry content.

In other words, the activities seek a productive synthesis of collaborative-discourse, shared-visualization, coconstruction, and argumentation skills applied in the domain of beginning geometry. They operationalize "deep conceptual learning" of mathematics in terms of these measurable outcomes:

1. The quality and quantity of significant mathematical discourse in collaborative interactions.

2. Group explorations of mathematical objects and representations, including noticing and wondering.

3. Constructions of shared mathematical objects with dependencies.

4. Explanations, justifications and proofs of conjectures proposed in chat interactions.

5. Engagement in significant mathematical discourse involving geometric notions of congruence, symmetry, dependencies, relationships, transformations and deduction.

\section{INCREASING SKILL LEVELS}

The set of activities should gradually increase student skill levels in each of the identified dimensions. The design starts out assuming relatively low skill levels and gradually increases the level of skill expected. Concomitant with this is a progressive shift from scaffolded instruction to open-ended inquiry.

1. The discourse begins with having students greet each other online and then negotiate about who will do what, when in the online environment. Students are next asked to comment on their noticings and wonderings. Later, they are to make conjectures. Finally, they are expected to explain things to each other, make sure that everyone understands, and produce presentations of group findings. Linguistic, conceptual and procedural skills developed in collaborative work eventually contribute to individual skills.

2. The exploration begins with being introduced to software widgets and tools. It goes on to increasingly complicated geometric drawings. Then, students are expected to construct geometric objects themselves and in small groups. Finally, they are given open-ended scenarios and encouraged to figure out how to explore unknown mathematical territory.

3. Construction skills gradually grow from dragging existing dynamic objects, to constructing with step-bystep instructions, to figuring out how to construct objects with specific dependencies, to defining their own custom construction tools, to constructing objects of their own design in open-ended micro-worlds. The skill level progresses from novice to a reasonable command of GeoGebra's geometry tools. A transition to GeoGebra's algebra connection (analytic geometry) is provided at the end, opening up GeoGebra's multiple representations of geometric diagrams, analyticgeometry graphs, spreadsheet data, 3-D transformations and a computer-algebra system.

4. Proof in geometry is introduced slowly, with a focus on noticing and wondering. This is followed by formulation of text-chat-based explanations and multimedia documentation of findings. The explanations gradually entail increased levels of justification, finally approaching formal proofs, without ever reaching the completely formalized version of routinized twocolumn proof.

5. The geometry content starts by covering many of the activities in Book I of Euclid's Elements (300 BCE/2002), but implemented in the computersupported collaborative-learning medium of multi-user dynamic geometry. It incorporates the beginning standards for high school geometry in the new Common Core Standards (CCSSI, 2011), including congruence, symmetry and rigid transformations. The fundamental features of triangles are examined first, and then 
students are encouraged to explore similar features for quadrilaterals. For instance, students are involved in designing hierarchies of kinds of triangles or quadrilaterals based on alternative representations and dependencies of congruence, symmetry and rigid transformations. Finally, a sampling of creative objects, micro-worlds and challenge problems are offered for student-centered exploration.

There is a theoretical basis for gradually increasing skill levels in terms of both understanding and proof in geometry. Here "understanding" and "proof" are taken in rather broad senses. The van Hiele theory (see deVilliers, 2003, p. 11) specifies several levels in the development of students' understanding of geometry, including:

1. Recognition: visual recognition of general appearance (something looks like a triangle).

2. Analysis: initial analysis of properties of figures and terminology for describing them.

3. Ordering: logical ordering of figures (a square is a kind of rectangle in the quadrilateral hierarchy).

4. Deduction: longer sequences of deduction; understanding of the role of axioms, theorems, proof.

The implication of van Hiele's theory is that students who are at a given level cannot properly grasp ideas presented at a higher level until they reach that higher level. Thus, a developmental series of activities pegged to the increasing sequence of levels is necessary to effectively present the content and concepts of geometry, such as, eventually, formal proof. Failure to lead students through this developmental process is likely to reinforce student feelings of inadequacy and consequent negative attitudes toward geometry.

Citing various mathematicians, deVilliers (2003) lists several roles and functions of proof, particularly when using dynamic-geometry environments:

1. Communication: proof as the transmission of mathematical knowledge.

2. Explanation: proof as providing insight into why something is true.

3. Discovery: proof as the discovery or invention of new results.

4. Verification: proof as concerned with the truth of a statement.

5. Intellectual challenge: proof as the selfrealization/fulfillment derived from constructing a proof.

6. Systematization: proof as the organization of various results into a deductive system of axioms, major concepts and theorems.

In his book, deVilliers suggests that students be introduced to proof by gradually going through this sequence of levels of successively more advanced roles of proof through a series of well-designed activities. In particular, the use of a dynamic-geometry environment can aid in moving students from the early stages of these sequences (recognition and communication) to the advanced levels (deduction and systematization). The use of dragging geometric objects to explore, analyze and support explanation can begin the developmental process. The design and construction of geometric objects with dependencies to help discover, order and verify relationships can further the process. The construction can initially be highly scaffolded by instructions and collaboration; then students can be guided to reflect upon and discuss the constructed dependencies; finally they can practice constructing objects with gradually reduced scaffolding.

This can bring students to a stage where they are ready for deduction and systematization that builds on their exploratory experiences. Furthermore, by working through the different roles of proof, math teachers and students are exposed to a richer conception of proof, in line with contemporary theories of proof, such as those by Lakatos (1976) and Livingston (1999).

\section{Discourse AND TECHNOLOGY ABOUT} DEPENDENCIES

The curricular activities center particularly on facilitating and supporting lessons in geometric dependency. GeoGebra allows one to construct systems of inter-dependent geometric objects. The dependencies built into dynamic-geometry constructions are intimately related to proofs illustrated by those constructions. Often, to understand a dependency and to be able to implement it in a construction is tantamount to being able to articulate a proof and to explore its validity dynamically (Stahl, 2012b). Students have to learn how to think in terms of these dependencies. They can learn through visualizations, manipulations, constructions and verbal articulations. These can all be modeled by examples, and these skills can be developed gradually.

Our concerns about fostering significant mathematical discourse are incorporated in this focus on dependency in geometry as follows:

1. Increase the ability of math teachers and students to engage in significant mathematical discourse about geometric dependencies.

2. Provide math teachers and students with a coherent sequence of activities exploring mathematical dependencies.

3. Empower math teachers and students to construct their own mathematical dependencies among objects in a dynamic-mathematics environment, which they can use in the future as well.

4. Increase the understanding of math teachers and students in why mathematical objects behave in the ways they are constrained to by their dependencies, possibly proving why the dependencies have specific consequences.

5. Increase the understanding of math teachers and students in the content of basic high-school geometry dependencies, including how to discuss them, explore them, visualize them, prove them and extend them.

We are now drafting and piloting versions of curricular activities designed to develop significant mathematical 
discourse focused on dependencies among geometric objects. Concomitantly, we are implementing software support for teachers and students to explore the dependencies and assembling materials for professional development to prepare teachers to enact this curriculum with their students.

\section{Mathematical Discourse}

The view of mathematical understanding as a communications skill suggests the central role of mathematically significant discourse and collaborative group practices in the growth of the abilities of students as they move from level to level in geometric understanding and proof. The activities for VMT-with-GeoGebra should support increasing fluidity with mathematically significant discourse.

The set of activities is therefore designed to:

1. Increase the ability of math teachers and students to engage in significant mathematical discourse.

2. Provide math teachers and students with a coherent sequence of activities exploring mathematical relationships and representations.

3. Empower math teachers and students to construct their own mathematical objects in a dynamic-mathematics environment, which they can use in their future study as well.

4. Increase the understanding of math teachers and students in why mathematical objects behave in the ways they do, possibly proving why they do.

5. Increase the understanding of math teachers and students in the content of basic high-school geometry content, including how to discuss, explore, visualize, prove and extend it.

The set of activities is designed to provide an educational experience in basic geometry to math teachers and students, taking them from a possibly novice level to a more skilled level, from which they can proceed more effectively without such designed activities. It is hoped that by providing activities on different levels for each of the dimensions, it can help most math teachers and students to increase their relevant skills - probably in quite different ways for different people.

Our design work is guided by socio-technical implications of continuing pilot studies as the technology and pedagogy of our project co-evolve. We are countering the problems that caused technical and cognitive distractions in our pilot studies by improving the software and testing the curriculum. The curriculum integrates tutorials about using the VMT and GeoGebra interfaces with carefully structured dynamic-geometry activities for virtual math teams. The activities systematically build up the background knowledge, group practices and problem-solving orientation needed for engaging in significant mathematical discourse.

\section{REFERENCES}

[1] Çakır, M. P., \& Stahl, G. (2012). The integration of mathematics discourse, graphical reasoning and symbolic expression by a virtual math team. In D. Martinovic, V. Freiman \& Z. Karadag (Eds.), Visual mathematics and cyberlearning. New York, NY: Springer. Web: http://GerryStahl.net/pub/visualmath.pdf.

[2] Çakir, M. P., Zemel, A., \& Stahl, G. (2009). The joint organization of interaction within a multimodal CSCL medium. International Journal of Computer-Supported Collaborative Learning. 4(2), 115-149. Web: http://GerryStahl.net/pub/ijCSCL 42 1.pdf.

[3] CCSSI. (2011). High school -- geometry. In Common Core State Standards Initiative (Ed.), Common core state standards for mathematics. (pp. 74-78).

[4] deVilliers, M. (2003). Rethinking proof with the Geometer's Sketchpad. Emeryville, CA: Key Curriculum Press.

[5] Euclid. (300 BCE/2002). Euclid's elements (T. L. Heath, Trans.). Santa Fe, NM: Green Lion Press.

[6] Lakatos, I. (1976). Proofs and refutations: The logic of mathematical discovery. Cambridge, UK: Cambridge University Press.

[7] Livingston, E. (1999). Cultures of proving. Social Studies of Science. 29(6), 867-888.

[8] Sfard, A. (2008). Thinking as communicating: Human development, the growth of discourses and mathematizing. Cambridge, UK: Cambridge University Press.

[9] Stahl, G. (2006). Group cognition: Computer support for building collaborative knowledge. Cambridge, MA: MIT Press.

[10] Stahl, G. (2008). Thinking as communicating: Human development, the growth of discourses and mathematizing. International Journal of Computer-Supported Collaborative Learning. 3(3), 361-368. Web: http://GerryStahl.net/pub/Sfardreview.pdf.

[11] Stahl, G. (2009). Studying virtual math teams. New York, NY: Springer.

[12] Stahl, G. (2012a). Dynamic-geometry activities with GeoGebra for virtual math teams. Web: http://GerryStahl.net/pub/activities.pdf.

[13] Stahl, G. (2012b). Translating euclid: Liberating the cognitive potential of collaborative dynamic geometry. Web: http://GerryStahl.net/pub/translating_euclid.pdf.

[14] Stahl, G. (2013). Theories of collaborative cognition: Foundations for CSCL and CSCW together. In S. Goggins \& I. Jahnke (Eds.), CSCL@work. (Vol. \#13 Springer CSCL Book Series). New York, NY: Springer. Web: http://GerryStahl.net/pub/collabcognition.pdf.

[15] Stahl, G., Ou, J. X., Weusijana, B. K., Çakir, M. P., \& Weimar, S. (2010). Multi-user GeoGebra for virtual math teams. GeoGebra: The New Language For The Third Millennium. 1(1), 117-126. Web: http://GerryStahl.net/pub/geogebra romania.pdf. 